\title{
A New Statistical Descriptor for the Physical Characterization and 3D Reconstruction of Heterogeneous Materials
}

\author{
Amirhossein Bagherian ${ }^{1}$, Seyedfarzad Famouri ${ }^{2}$, Mostafa Baghani ${ }^{1}$, Daniel George ${ }^{3 *}$, \\ Azadeh Sheidaei ${ }^{4}$ and Majid Baniassadi ${ }^{1,3 *}$ \\ ${ }^{1}$ School of Mechanical Engineering, College of Engineering, University of Tehran, Tehran, Iran. \\ ${ }^{2}$ The Robotic Surgery Lab, Mechanical Engineering Department, Concordia University, Montreal, QC, Canada \\ ${ }^{3}$ University of Strasbourg, CNRS, ICube Laboratory, Strasbourg, France \\ ${ }^{4}$ Aerospace Engineering Department, Iowa State University, Ames, IA 50011, United States
}

\begin{abstract}
$3 \mathrm{D}$ reconstruction of heterogeneous materials from 2D images is essential for a precise characterization of their physical properties (mechanical, thermal, electrical and so on). For this, statistical descriptors such as two-point correlation function (TPCF), lineal path function (LPF), or two-point correlation cluster function (TPCCF) are frequently used. But the effective properties of the reconstructed microstructures are not always corresponding to the real ones as the statistical distribution functions may distribute the material microstructure in a different way from the original one. This is more pronounced for cellular and porous materials such as trabecular bone, fuel cell, and rocks where the connectivity between clusters is not well correlated to the one of real material and degrades the materials physical behavior predictions. This paper proposes a new statistical descriptor, called Quality of Connection function (QCF), able to determine the quality of connections between clusters and has detailed statistical information about the microstructure distribution. The proposed descriptor is tested on trabecular bone obtained from X-ray micro-computed tomography, and used as example of heterogeneous material having a complex microstructure. Effective properties such as Young Modulus were calculated for these microstructures and compared with real ones. The new descriptor shows improved capacity to describe the material microstructure distribution and prediction of its physical properties.
\end{abstract}

Keywords: Two-Point Correlation Function, Two-Point Cluster Function, Statistical Descriptor, Quality of Connection Function, Heterogeneous Material

\section{Introduction}

The specific properties and behaviors of heterogeneous materials, particularly for biological materials, are greatly dependent on their microstructures [Torquato 2002; Buryachenko 2007; Nikolov 2010 ; Abdel Rahman et al. 2012]. When local behavior is unknown which is often the case, it lives most of the time only analyses to a coarse continuum behavior [Scala et al. 2016; Madeo et al. 2013]. Thus, the 3D modeling of these microstructures is required for a better precise

* Corresponding author 1: Majid Baniassadi, Email: m.baniassadi@ut.ac.ir

\footnotetext{
* Corresponding author 2 : Daniel George

Email: george@unistra.fr
} 
comprehension of the link between the overall performance and the microstructural characteristics (e.g. to estimate good transmission of mechanical and physical properties between the micro and the macro scale) [Kazempour et al. 2019; Bagherian et al. 2019; Rémond et al. 2016; Torquato and Haslach 2002].

A representation of heterogeneous materials may be done using statistical descriptors, considered as one of the most powerful tools integrating reconstruction and homogenization processes [Torquato and Haslach 2002; Baniassadi et al. 2011a; Hasanabadi et al. 2016]. Statistical continuum mechanics is also used to explain the structural morphology of materials via statistical correlation function [Izadi et al. 2017]. Hence, the effective properties of heterogeneous materials can be defined by the use of these correlation functions [Izadi et al. 2017; Fullwood et al. 2008]. For example, N-point correlation functions can be used to determine the mechanical, thermal, and physical properties of heterogeneous systems such as galaxies [Fullwood et al. 2010; Baniassadi et al. 2012; Hamedani et al. 2011].

The reconstruction of 3D models from a limited number of microstructural data generated from 2D cross sections is a classic inverse problem in a variety of applications for efficient design of the material, statistical analysis, and visualization. In the domain of material science, petroleum engineering, biology, and medicine, most of the time, only 2D images are accessible [Sheidaei et al. 2019; Rémond et al. 2016; Torquato and Haslach 2002, and Jiao et al. 2008]. But the reconstruction of 3D models based on stitching digitized serial section images, generated from imaging modalities such as Focused Ion Beam-Scanning Electron Microscope, X-ray computed tomography, scanning laser confocal microscopy and so on, are not genuinely appropriate for engineering application because of prohibitive technologies, lack of skilled operators and other numerous related issues. In consequence, the demand for credible alternative computational methods for the aforesaid 3D microstructural reconstruction has increased recently in various fields relevant to material sciences. A successful reconstruction procedure can supply non-destructive and low-cost means of estimating the macroscopic properties of heterogeneous materials [Sheidaei et al. 2013; Lim et al. 2018; Gigliotti et al. 2018; Rezaei et al. 2019] having complex internal microstructures such as bone microstructures for instance.

To reconstruct 3D microstructure from 2D images, two-point correlation function (TPCF), lineal path function (LPF), and two-point correlation cluster function (TPCCF) are frequently used [Lu and Torquato 1992; Lu and Torquato 1993; Torquato et al. 1988]. These descriptors are usually valuable tools when the material's microstructure is "well distributed" (close to homogeneous statistical distribution) and provide powerful tools to study phenomena such as percolation fluid flow. However, the variability of the microstructure distribution is dependent on the size of the representative volume element (RVE) for which the study is made. They will tend to work well for RVE with homogeneous statistical distribution, but will have difficulties to reproduce heterogeneous statistical ones (such as clustering). Hence, the dependent physical properties will be altered. To solve this, the development of an efficient descriptor able to highlight the variability of the local microstructures distribution might help to reach an efficient reconstruction procedure and generate a 3D microstructure with similar effective properties compared to the real one.

In this study, we use trabecular bone as an example of heterogeneous material having a complex heterogeneous microstructure. X-ray micro-computed tomography of the real trabecular bone microstructures was used for the initial microstructures. The new statistical descriptor is introduced and formulated with a computational framework. The capability of the approach is shown by comparing the effective properties of different types of bone microstructures. The new descriptor was applied to compute the effective properties such as Young modulus of the heterogeneous 
materials. The current study summarizes a series of numerical case studies to illustrate the computational efficiency and capabilities of this new descriptor.

\section{Method}

\subsection{Definition of statistical descriptors}

Correlation functions are useful descriptors for multiphase heterogeneous materials, which mainly require rigorous method of characterization of the microstructure [Torquato and Haslach 2002]. For a material with a given microstructure, the function of phases is defined as,

$$
x_{s}^{n}= \begin{cases}1 & s \text { in phase } n \\ 0 & \text { otherwise }\end{cases}
$$

Where $n$ and $s$ are the intended phase and position, respectively, for Eigen-microstructure. The grid points are numbered from 0 to $S-1$. More particularly, for a two-phase media, the phases are specified with $n$ and $n^{\prime}$. Two-point correlation functions (TPCFs) is the first descriptor that can obtain a geometrical specifications of heterogeneous materials (see Fig. 1).

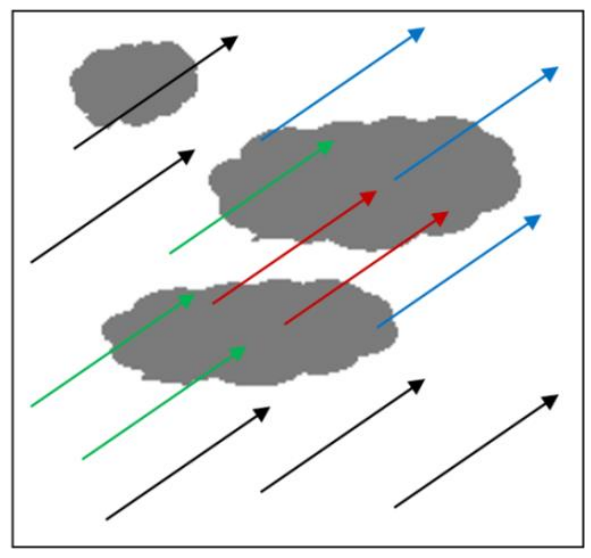

Fig. 1. Schematic representation of possible TPCFs for a two-phase material microstructure.

Moreover, TPCF is defined beneath as:

$$
c_{2}^{n n^{\prime}}(r)=\frac{1}{S} \sum_{s=0}^{S-1} x_{s}^{n} x_{s+r}^{n^{\prime}}
$$

where $r$ is the correlation vector defined in terms of relative differences between the position vectors of the different voxels of the structure.

TPCFs can be calculated using the Fast Fourier Transform (FFT) of the microstructure functions for periodic microstructures and it can be solved by using the convolution defined as :

$$
C_{k}^{n n^{\prime}}=\mathcal{F}\left(c_{2}^{n n^{\prime}}(r)\right)=\frac{1}{S}\left|X_{k}^{n}\right| e^{i \theta_{k}^{n}}\left|X_{k}^{n^{\prime}}\right| e^{-i \theta_{k}^{n^{\prime}}}
$$

One limit of the Two-point correlation function is that it correlates only the states of the starting and the ending points of the correlation vector. In addition, the connection between these two states is not accounted for in the calculation. Therefore, when the connection between the states is required, other statistical descriptors should be defined, such as the Two-point cluster function or lineal path function. The two-point cluster function (TPCCF) is the probability of finding the starting and 
ending points of a correlation vector in the same cluster and state. This function requires more detailed signatures of the microstructure compared to the TPCF. However, this descriptor cannot reflect specific cluster connectivity between phases (such as thin long connectivity between two clusters) that will define the quality of connections between the states. Lineal-path function (L(r)) is useful for differentiating domains; it can estimate the probability of finding a line segment with a given length which is entirely inside the target state. It is known to be a better microstructure descriptor than TPCF.However, these different tools have not showed good results for the particular structure of bone and cannot be a good signature for these microstructures [Torquato and Haslach 2002; Jiao et al. 2009]. Hence, in this research, a new statistical descriptor is introduced, which can reflect a better signature for particular microstructures such as bone.

\subsection{New statistical descriptor description}

The new statistical descriptor, named Quality of Connections Function (QCF), is based on the idea that the distribution of phases in the microstructure of multiphase materials affects their physical properties. Statistical functions usually measure a specific feature of the materials and correlate it with their properties like strength and stiffness for example. The new descriptor provides a quantification of the geometrical connection quality in the structure and gives detailed statistical information about the phase's connectivity in the microstructure. The volume fraction is not integrated in QCF which prevents a direct evaluation of the corresponding physical properties of the material. The term quality expresses how different parts of one phase connect to each other in a multiphase material. To explain the term quality measured in the microstructures, we need to introduce two parameters in the multiphase materials; (i) the shortest distance (SD) which is the linear length connecting two nodes in space, and (ii) the shortest path (SP) which is the length of the path between the same two nodes but going only through the same phase (see Fig. 2).

We first calculate the shortest path (SP) within the same material phase and the shortest distance (SD) between two voxels (in 3D) or two pixels (in 2D) of the microstructure. By dividing SD by SP we have a parameter that varies between zero and one. When this fraction is equal to one, it means that the shortest distance is equal to the shortest path. That means the line which connects two nodes of a phase is fully in the same phase. This indicates a good connection between two points. As the ratio $\mathrm{SD} / \mathrm{SP}$ is decreasing towards zero, the quality of this connection gets poorer and poorer in the sense that the path to reach the two points inside the same phase becomes more tortuous and longer up to the point it becomes impossible.

In essence, QCF is related to percolation through the tortuosity of the microstructure network $\tau=\frac{L}{R}$ as defined by [Shanti et al. 2014]. Here $\tau$ is the tortuosity, $L$ is the geodesic length of a pore channel, and $\mathrm{R}$ is the end to end length of the pore channel. However, the classical use of percolation theory (for fluid flow or capillarity analysis through porous media) comes through standard homogenization procedures as defined for example by [Bazarin 2021; Marafani 2020; Hilfer 2002; Gerrard 2010] to study the effective behavior through periodic, stochastic or homologous microstructure distribution. Hence, although these methods include the local microstructure distribution effects, the homogenization process will "average" the physical properties over the studied sample. The current QCF descriptor aims to extract information at the scale of the microstructure, even by remaining a statistical descriptor and keep the geometrical distribution information even at the effective length. This will enable improved 3D reconstruction by integrating these aspects of local clustering scenario.

For any given microstructure distribution, a QCF graph can be plotted from the calculation of the quality of connections at each vector length. QCF is calculated for all vectors that are fully placed 
inside the microstructure and both ends of the vector are in the same phase. The average of this value for each vector length is the QCF value related to that length. For an anisotropic microstructure, mechanical properties vary in each direction. Hence, the curve of QCF must also be different.

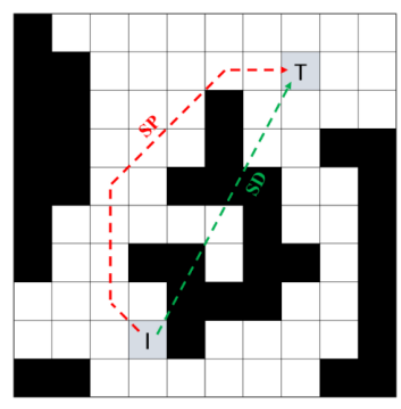

Fig. 2: Schematic definition of shortest distance (SD) and shortest path (SP) between two random nodes in a 2D two-phase microstructure

A vector is considered valid when fully placed inside the microstructure and both of its ends located in the same phase. If both ends are in the same cluster, there is a path between two nodes. Else, SP is infinite and SD/SP is equal to zero. Fig. 3 shows some of applicable vectors in the horizontal direction. The black phase represents the solid phase of bone microstructure (trabecular bone) and the white phase is the bone marrow. The eligible (valid) vectors are represented by the green arrows. On the contrary, invalid vectors are in red, meaning that no path exists within the same phase between both ends of these vectors.

For validation purposes, we consider here only a 2D case where in-plane connections will be evaluated. To calculate the QCF value of each vector length, the parameters SP and SD, and fraction $\mathrm{SD} / \mathrm{SP}$ are calculated for each vector. Then the average value for the fraction SD/SP, calculated for all vector at one given length, is extracted. This corresponds to the QCF value of this microstructure at that given length. When calculating this QCF parameter for all vector lengths between 0 and the microstructure total length, a graph is obtained as a result of the descriptor for this given microstructure.

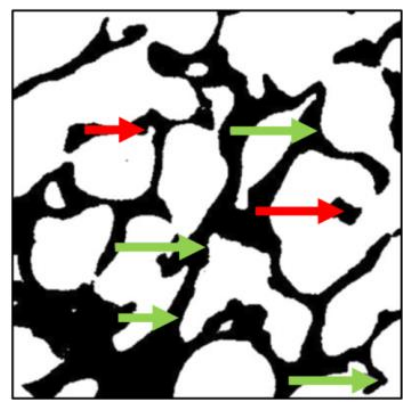

Fig. 3: Heterogeneous microstructure distribution such as trabecular bone. Red arrows indicate there is no path between two nodes of the microstructure; green arrows indicate both nodes are in the same cluster phase.

The most complicated and time-consuming part of this analysis is to find the shortest path between two nodes in a multiphase material, as the shortest path between an initial and a target node is the length crossing through the same phase. Navigation and finding of the shortest path to a goal location is one of the fundamental problems in path planning. There are many approaches to this problem. A common approach is the A-Star algorithm [Premakumar 2016]. It will be used to calculate the parameter SP in this work. A-Star algorithm process uses the distance between the current location and the target and moves to the node that has the smallest distance. It evaluates all possible nodes in the neighborhood by combining $h(n)$, the distance (cost) to the ongoing node and $g(n)$, the distance (cost) from that node to the goal node. The total cost $f(n)=g(n)+h(n)$ is calculated for each successor node and the one with the smallest cost of $f(n)$ is selected as a successor. This process 
continues till the robot reaches the target node (if possible). Routes ending in a dead end assume to have infinite cost function and are not considered. The dead end means the marker is surrounded by voxels from the other phase and there is no way to go forward. When the robot ends up in a dead end, it explores alternate routes. This algorithm tests all the possible routes from the initial node to the target node. Finally, the path that connects the initial node to the target node with the lowest cost is chosen as the shortest path between these two nodes going through the same phase.

\section{Results and discussion}

QCF analyses were carried out on bone microstructures. A complete interpretation of the results is provided, presenting the capability and advantages of QCF over other statistical descriptors. First, the accuracy and validity of QCF in the assessment of the microstructures is evaluated. Next, a comparison between QCF and existing descriptors is made. The abilities of the different descriptors on several different microstructures were examined. Finally, the results will present the best performances of the descriptors to estimate the effective stiffness of the microstructures.

\subsection{Analysis and comparison of QCF for similar microstructures}

To examine the quality of connections for two-phase microstructures, four cases were considered in two groups. Each group contains a pair of analogous microstructures. According to the information presented in Table 1, volume fraction, number of clusters of hard phase, and the volume fraction of the largest cluster of hard phases are defined.

\begin{tabular}{|c|c|c|c|c|}
\hline \multicolumn{5}{|l|}{ Model } \\
\hline $\begin{array}{l}\text { Global volume } \\
\text { fraction VF }\end{array}$ & 0.2522 & 0.2564 & 0.3271 & 0.3178 \\
\hline Number of clusters & 18 & 10 & 5 & 7 \\
\hline Largest cluster VF & 0.1577 & 0.1663 & 0.3215 & 0.2979 \\
\hline
\end{tabular}

Table 1: Definition of the four (2 times 2) validation test cases. Volume fraction, number of clusters of hard phase, and the volume fraction of the largest cluster for each microstructure is given. Each microstructure has a side dimension of $1 \mathrm{~mm}$ by $1 \mathrm{~mm}$ length.

The term cluster defines a group of pixels of one phase stuck together. There might be one or more clusters of a phase in a multiphase material. In the first group (model one and two), the overall volume fractions are almost identical but there is no obvious large cluster although the sizes of the largest cluster are not too different. The second pair (models three and four) contains each a large cluster for which the volume fraction is close. The microstructures are composed of two phases, hard and soft. The Young modulus assigned for hard and soft phases are $15 \mathrm{GPa}$ and $1 \mathrm{KPa}$, respectively. Poisson's ratio for both phases is 0.3 . The QCF are evaluated for all four microstructures in the two main directions, vertical and horizontal. 
Fig. 4 shows the QCF plots for models one and two and Fig. 5 shows the results for models three and four. Horizontal and vertical axes show the length of vectors in $\mu \mathrm{m}$ and values of QCF, respectively. Fig. 4a shows higher QCF values for case 1 (blue) in the horizontal direction compared to case 2 (red). Fig. 4b clearly shows higher QCF value over the entire range of correlation lengths for case 2 (red) in the vertical direction compared with case 1 (blue).
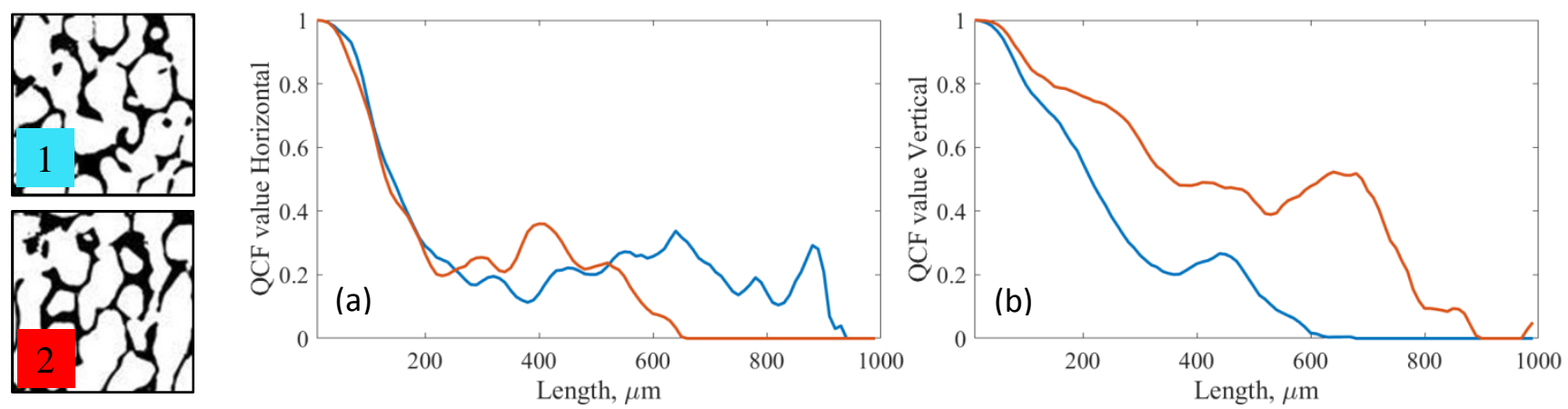

Fig. 4: Distributions of the quality of connection functions (QCF) for cases number 1 and 2 in both vertical and horizontal directions. These curves show the influence of microstructure distribution (as per the localization of higher density clusters) within each sample in the two horizontal and vertical directions.

In the second group (cases 3 and 4), QCF distributions are very similar in the vertical direction (Fig. 5b). However, in the horizontal direction (Fig. 5a), case number 3 (blue) shows a gradually decreasing trend, while in the other case 4 (red), the trend shows sudden drops. The first drop occurs in the range between $0 \mu \mathrm{m}$ and $200 \mu \mathrm{m}$ and the second drop happens between $500 \mu \mathrm{m}$ and $800 \mu \mathrm{m}$.
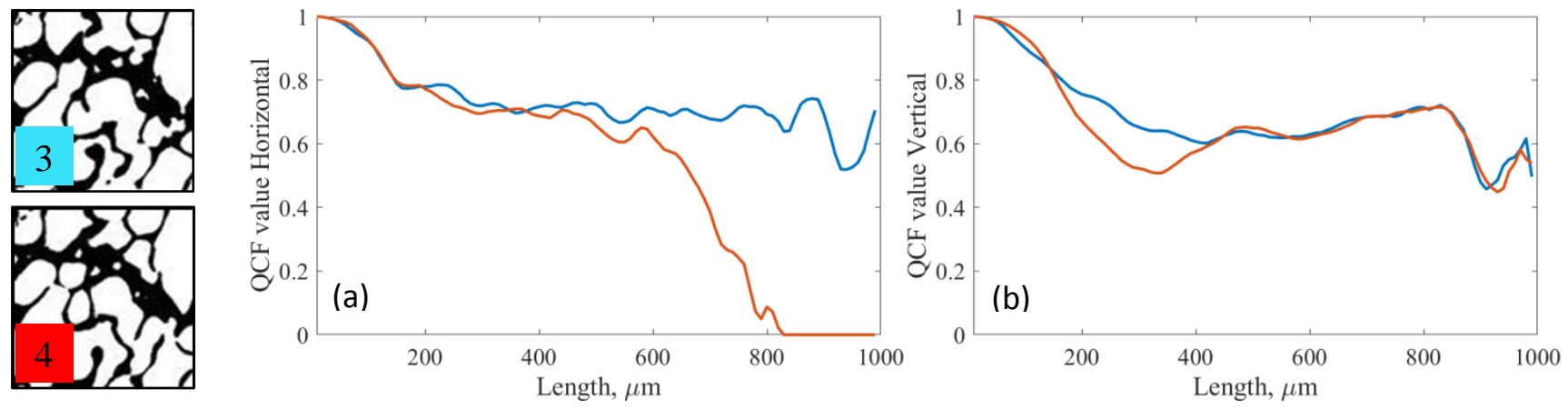

Fig. 5: Distributions of the quality of connection functions (QCF) for cases number 3 and 4 in both vertical and horizontal directions.

We hypothesize that in microstructures with similar volume fractions but with different quality of connections, the one that shows the higher QCF value of connection has a higher strength. Hence, under this hypothesis, it is likely that case number 3 (blue) has a stronger structure (higher stiffness) in the horizontal direction since it shows higher values of QCF in this direction. To test this hypothesis, finite element analyses were carried out. We assumed overall macroscopic linear elastic behavior under small strain theory (as for bone material is under linear elastic behavior) and any sufficiently small applied mechanical load will provide the overall force-displacement curve from which Young's modulus can be extracted. Young moduli of microstructures in both directions were calculated from simple compression tests. 


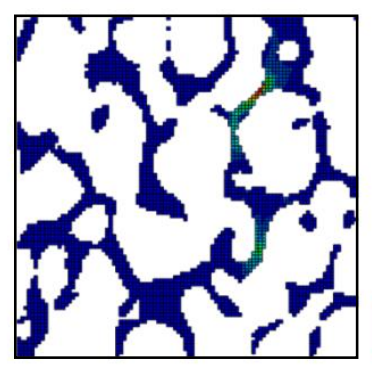

Young Modulus

(Horizontal)

$=0.00799 \mathrm{MPa}$

Young Modulus

(Vertical)

$=0.00844 \mathrm{MPa}$

1

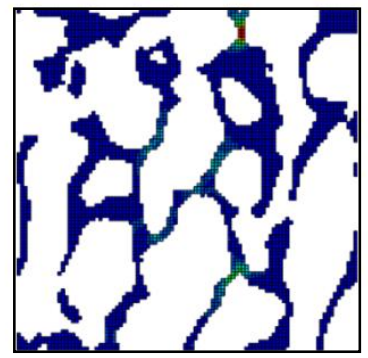

(Horizontal)

Young Modulus

(Vertical)

$=3.1 \mathrm{MPa}$

2

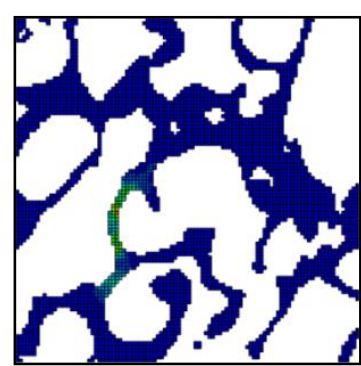

Young Modulus

(Horizontal)

$=4.5 \mathrm{MPa}$

Young Modulus

(Vertical)

$=17 \mathrm{MPa}$

3

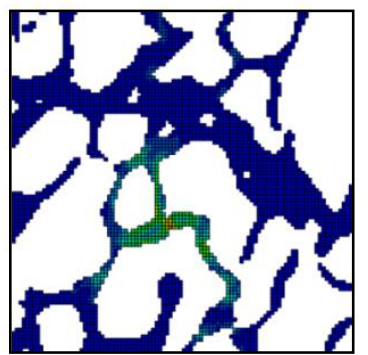

Young Modulus

(Horizontal)

$=0.00944 \mathrm{MPa}$

$=0.00768 \mathrm{MPa}$

Fig. 6: Localization of Von Mises stress distributions for all four cases under simple compression (horizontal then vertical) on hard voxels (trabecular phase). Young's moduli of each microstructure in both horizontal and vertical directions are calculated for all cases.

As it was expected, the results presented in Fig. 6 support the proposed hypothesis that higher QCF value presents a higher stiffness with higher QCF value. Higher Young modulus values (Fig. 6) are presented for each structure that have higher QCF values (Fig. 4 and Fig. 5).

\subsection{Comparison of statistical descriptors}

Descriptors measure different parameters in a microstructure to estimate their physical properties. In order to show the capability of the different descriptors to estimate these, a group of eight different microstructures were used (see Fig. 7 left, $\mathrm{N}^{\circ} 1$ to 8). The four statistical descriptors, QCF (Quality of Connections Function), TPCF (Two-Point correlation Function), TPCCF (Two-Point Correlation Cluster Function), and Lineal Path Function (LPF) were calculated for all cases. The cases are divided into four groups considering their similar physical characteristics (see Table 2).

\begin{tabular}{|c|c|c|c|c|c|}
\hline Model & $\begin{array}{c}\text { Global } \\
\text { VF }\end{array}$ & $\begin{array}{c}\text { Number of } \\
\text { Clusters }\end{array}$ & $\begin{array}{c}\text { Biggest Cluster } \\
\text { VF }\end{array}$ & $\begin{array}{c}\text { Young Modulus } \\
\text { Horizontal (MPa) }\end{array}$ & $\begin{array}{c}\text { Young Modulus } \\
\text { Vertical (MPa) }\end{array}$ \\
\hline 1 & 0.2915 & 10 & 0.2126 & 0.198 & 16.9 \\
\hline 2 & 0.3059 & 8 & 0.2779 & 22.8 & 33.2 \\
\hline 3 & 0.3178 & 7 & 0.2979 & 0.00944 & 19.9 \\
\hline 4 & 0.3436 & 7 & 0.3056 & 58 & 0.00812 \\
\hline 5 & 0.2408 & 11 & 0.209 & 5.7 & 0.0073 \\
\hline 6 & 0.358 & 14 & 0.2018 & 0.2798 & 104.6 \\
\hline 7 & 0.3623 & 9 & 0.3309 & 0.0094 & 476.8 \\
\hline 8 & 0.3603 & 5 & 0.3548 & 17.7 & 18.1 \\
\hline
\end{tabular}

Table 2: Description of the physical characteristics of the eight tests case presented in Fig. 7 left; global and largest cluster volume fractions, number of clusters of hard phase, and Young modulus calculated from QCF descriptor for each of the eight microstructures. 


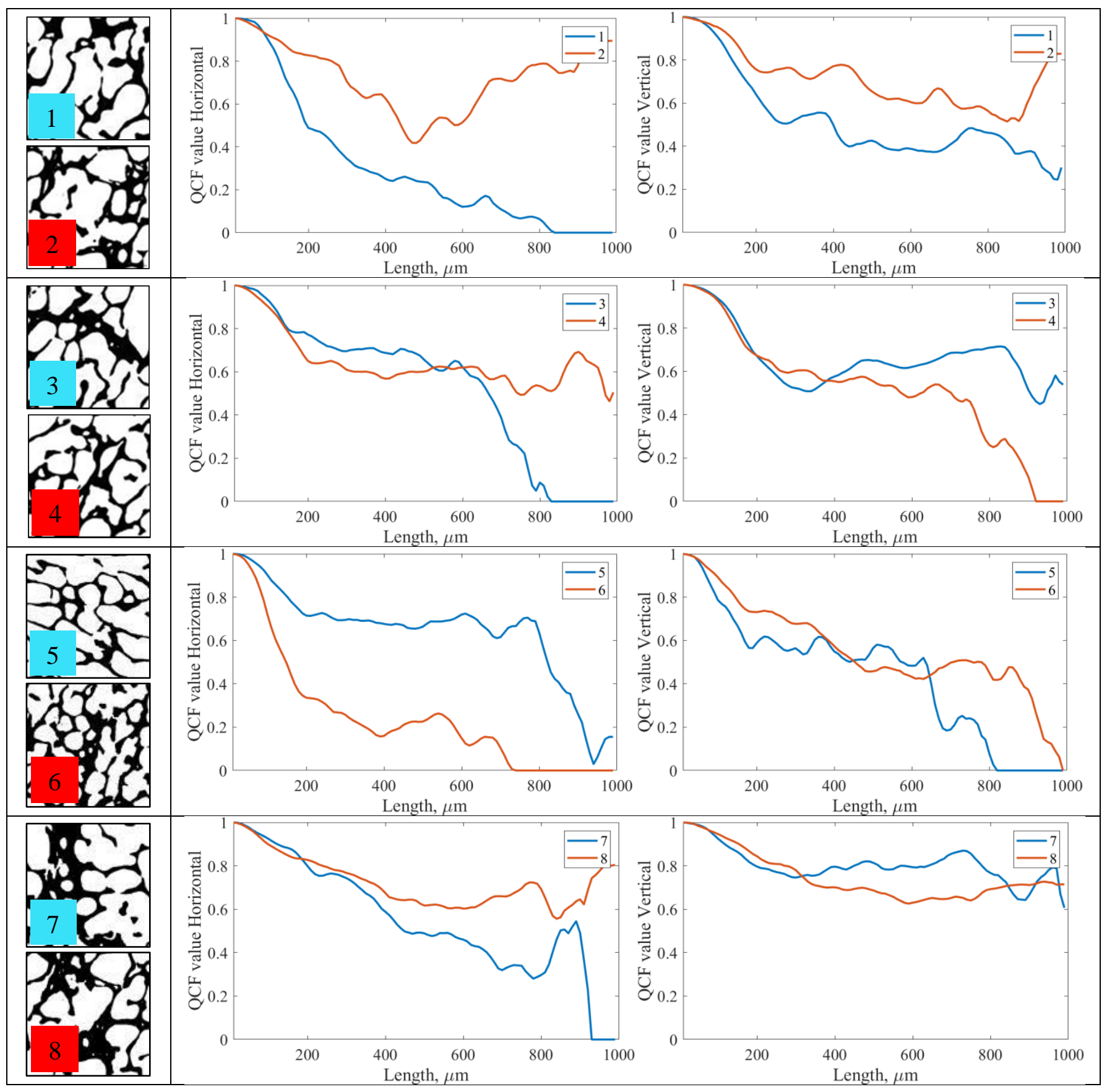

Fig. 7: Left shows the four cases (number 1 to 4) to compare the different descriptors. Each sample has a $1 \mathrm{~mm}$ by $1 \mathrm{~mm}$ side length. Right shows the corresponding QCF distributions of the four cases in both horizontal and vertical directions. Global volume fraction of case 1 and 2 are similar whereas biggest cluster volume fraction of case 3 and 4 are similar (see Table 2).

In the first and fourth group (Model 1-2 and 7-8), we have close global volume fraction while in the second and third group (Model 3-4 and 5-6), the volume fractions of the largest cluster of hard phases are almost equal. The QCF plots for these four cases are presented in Fig.7 right. The results of the comparison of other classical descriptors are presented in Fig.8. 

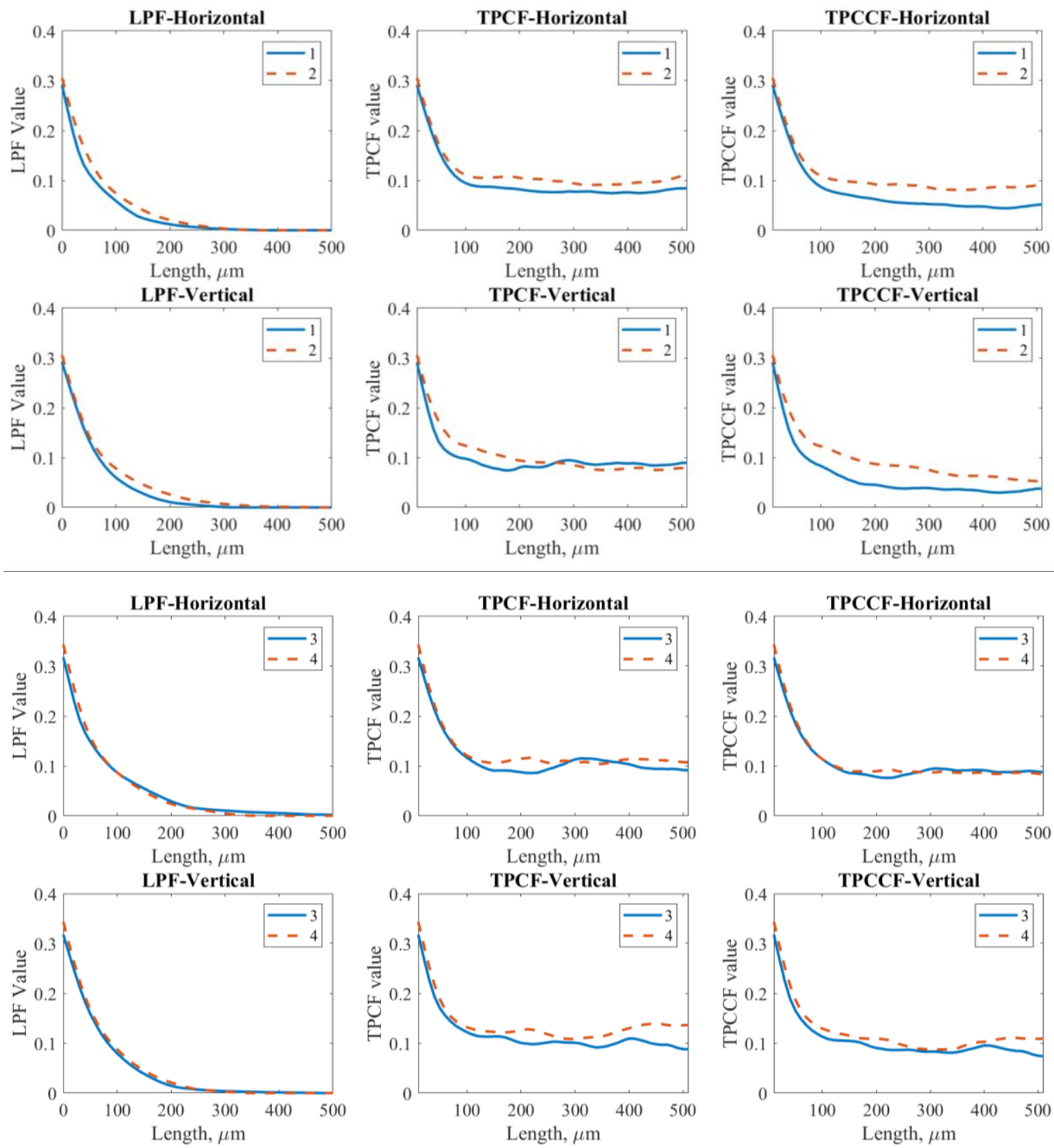

Fig. 8a: Classical statistical LPF, TPCF, and TPCCF descriptors distributions for cases 1 to 4 presented in Fig. 7 left. Rows one and two, show the results of the descriptor comparisons between cases 1 and 2. Rows three and four show the results of the descriptor comparisons between cases 3 and 4.

The results of the comparison of classical descriptors showed no large difference in the horizontal and vertical directions while the results of the QCF descriptor are very different. Hence, no conclusion can be drawn from the distribution of the classical descriptors regarding the physical properties of the microstructures such as Young's modulus. However, from the QCF distributions, a large variation of Young's modulus for all cases can be extracted as presented in Table 2, but not visible on the other descriptors. Fig. 8 seems to show that there is an increase of sensitivity between the descriptors from LPF, TPCF, and TPCCF. TPCCF seems to be the most sensitive to the 
microstructure differences and gives a better understanding of their strength. However, none of them are really able to extract useful information about the physical characteristic.
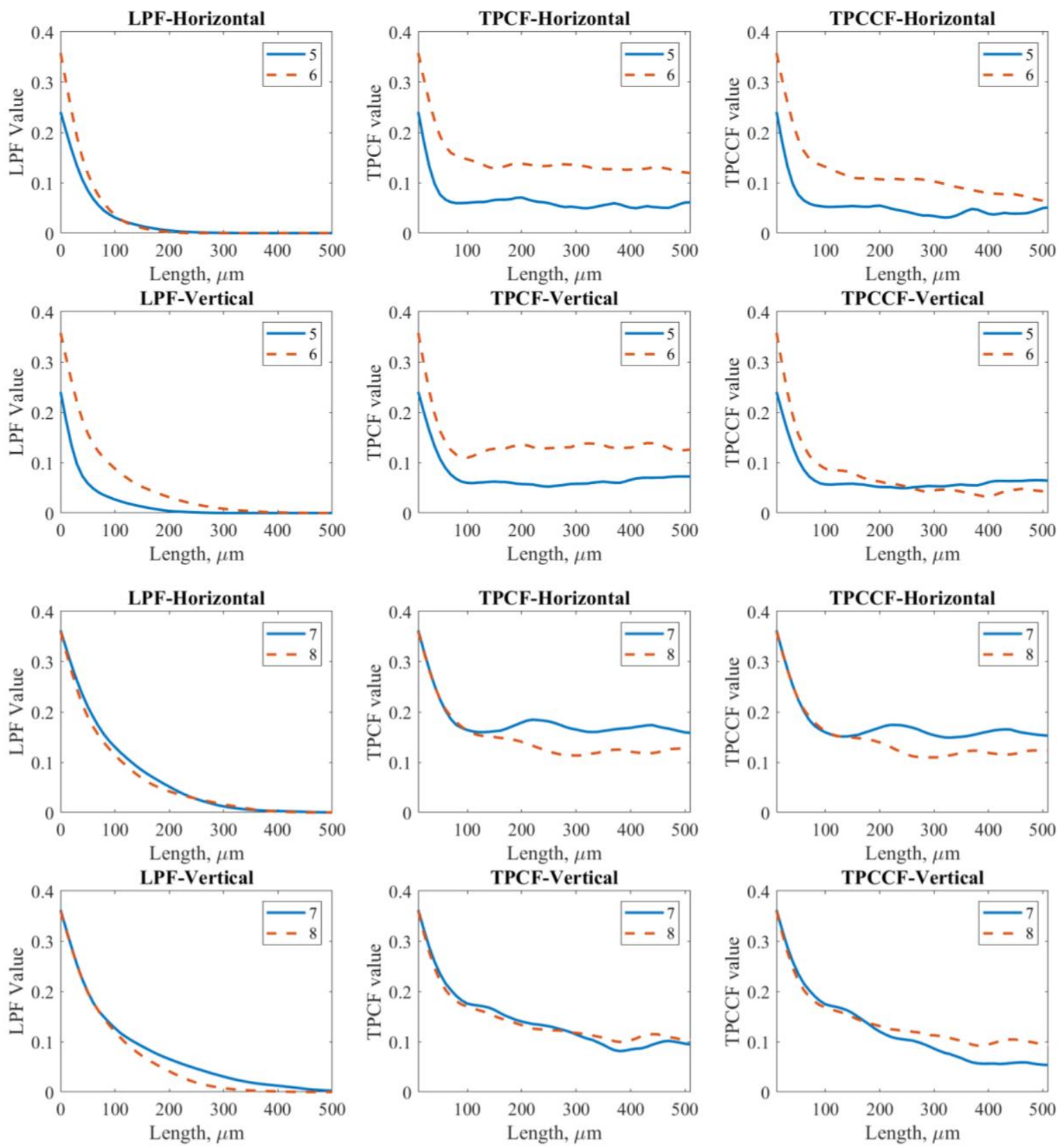

Fig. 8b: Classical statistical LPF, TPCF, and TPCCF descriptors distributions for cases 5 to 8 presented in Fig. 7 left. Rows one and two, show the results of the descriptor comparisons between cases 5 and 6. Rows three and four show the results of the descriptor comparisons between cases 7 and 8.

On the contrary, QCF gives very good information. Case 2 is stronger in both horizontal and vertical directions compared to case 1 (two cases with similar global volume fraction). This is clearly visible from the QCF trends of Fig. 7 right. For the two other cases 3 and 4, QCF describes the strength of the structure perfectly. In the vertical direction, case 3 has higher stiffness as the results show its Young modulus is higher (Table 2). However, in the horizontal direction, the quality of connections 
for case number 4 is better and shows no drop in the trend compared with case number 3 . In this direction, case 4 has a higher Young modulus (Table 2) compared to case 3. Overall, the QCF curve of the other four microstructures shows that in every comparison, when the QCF value of a microstructure has higher value in a given direction, this corresponds to higher stiffness in that direction.

\subsection{Effect of deletion of junctions}

In cellular material like trabecular bone, material degradation such as osteoporosis can lead to a considerable drop in the strength and stiffness of the bone microstructure that can be of important concern for the patient. It is therefore important to assess the capability of the QCF descriptor to detect this. The measurement of the missing junction can either come from a bad image acquisition (from the imaging techniques) or can be really present inside the microstructure and therefore it is essential to be able to quantify this effect. To test this and analyze the ability of the new descriptor in the comprehension of deletion of junctions, we arbitrarily deleted some "well chosen" connections within the real given bone microstructure causing a severe reduction in its stiffness and strength (Fig. 9) and run the new QCF descriptor to assess its capacity to detect such scenario.

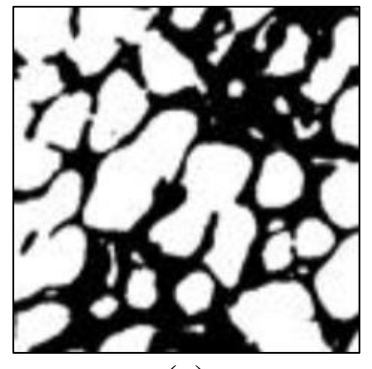

(a)

Young Modulus $($ vertical $)=641.6 \mathrm{MPa}$

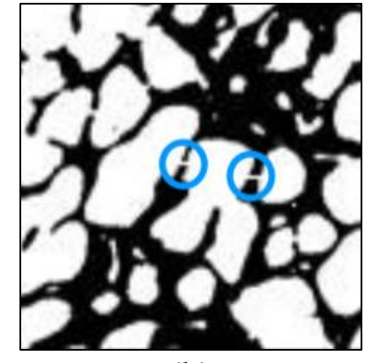

(b)

Young Modulus $($ vertical $)=220.3 \mathrm{MPa}$

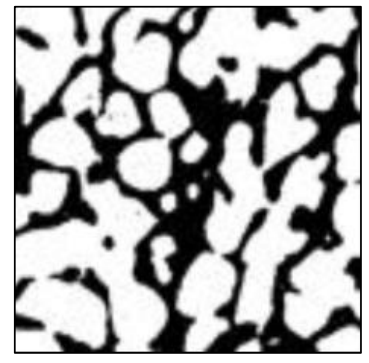

(c)

Young Modulus (horizontal) $=20.4 \mathrm{MPa}$

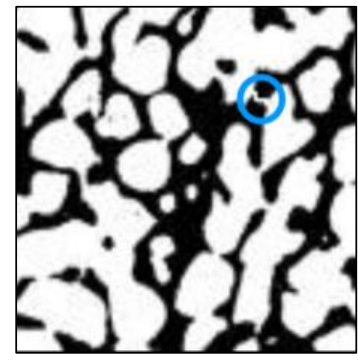

(d)

Young Modulus (horizontal) $=0.28 \mathrm{MPa}$

Fig. 9: Two different trabecular bone microstructures: (a) and (c) are initial microstructures, (b) and (d) after removing junctions. Each sample has a $1 \mathrm{~mm}$ by $1 \mathrm{~mm}$ side length.

For the evaluation of the Young's modulus for the two cases in Fig. 9a and Fig. 9b, we show the distribution of the four statistical descriptors in the vertical direction before and after the deletion of junctions in Fig. 10. When removing only a few (small number of) voxels of the microstructures, the classical statistical descriptors will not distinguish the change, and 3D microstructure reconstruction based on these descriptors will result in poor prediction of stiffness and strength (see Fig. 10 and Fig. 11). No difference is observed for the classical descriptors plots before and after the deletion of junctions. On the contrary, the QCF distribution drops after the deletion of the junctions which means these junctions were critical in the vertical direction of the microstructure. In Fig 9b, two junctions were removed; the vertical Young modulus decreased from 641.6 MPa to 220.3 MPa and in Fig 9d, the horizontal Young modulus decreased from 20.4 MPa to 0.28 MPa after removal of only one junction. 

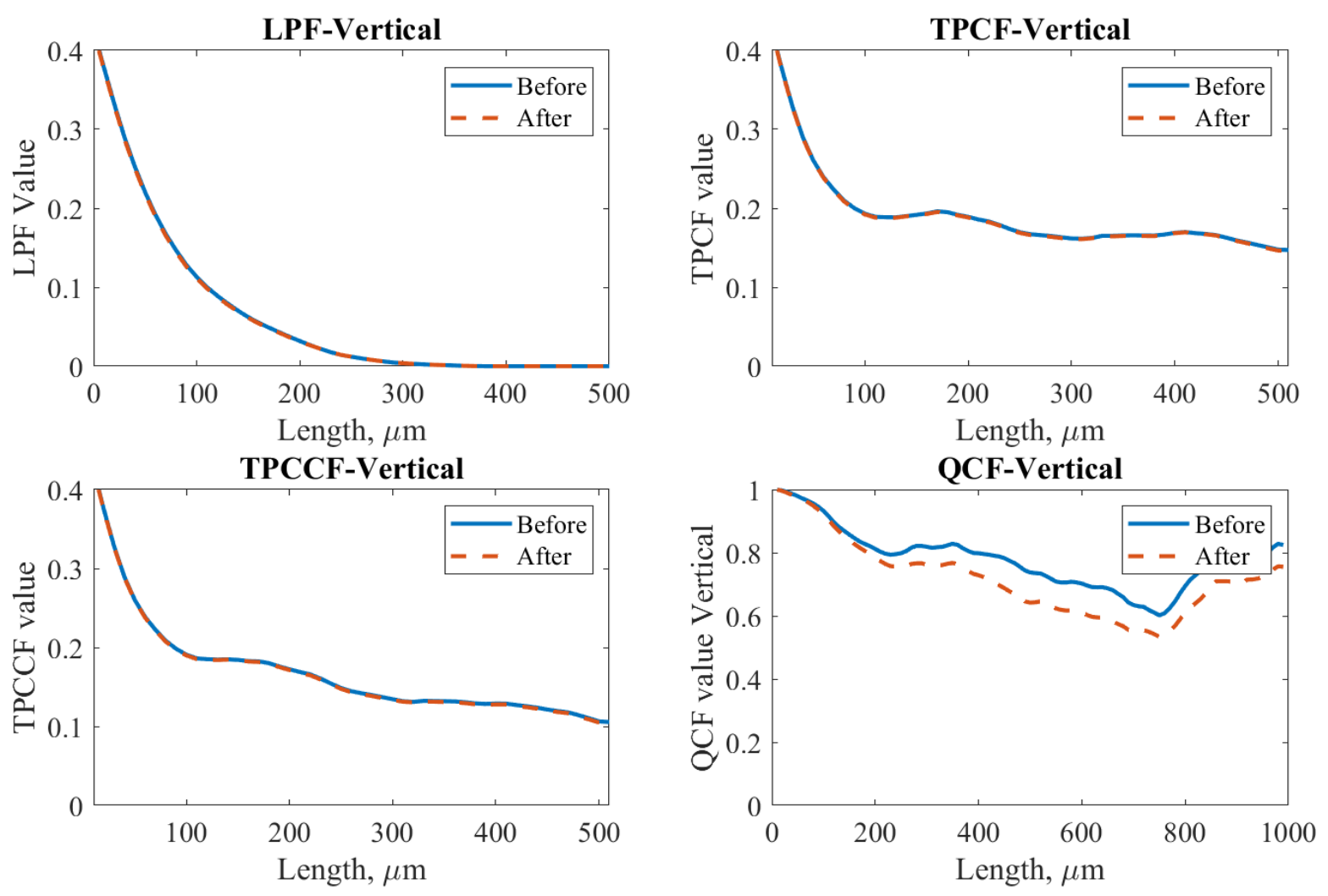

Fig. 10: Distribution of all descriptors in the vertical direction before and after deletion of junctions for Fig.9a and Fig 9b. For LPF, TPCF, and TPCCF, solid line shows the values for initial microstructure and dashed line shows the values after removing junctions. For QCF, the values after the deletion of junctions are shown with colored lines.

For case in Fig. 9c and Fig. 9d, the situation is almost identical and the classical descriptors show no sensitivity to the deletion of junctions. The results are presented in Fig. 11. The TPCCF descriptor was able to detect a small variation after creation of the gap since after deletion of the junction, a substantial part of the main cluster of hard phase split. This led to a small decline in the TPCCF plot trend. Without deletion of this particular junction and keeping the main cluster in one piece, the TPCCF would not see any variation and remain identical, even deleting another junction out of the main cluster. On the contrary, the QCF descriptor showed a large variation before and after deletion of the junction, even though it corresponds to only a very small variation of the bone density. This provides very important information regarding the calculation of the corresponding mechanical properties such as the Young's modulus. The QCF descriptor is able to show this variation where other descriptors were not. Hence using QCF descriptor will provide much better understanding of the corresponding physical properties of reconstructed materials compared to other classical descriptors such as TPCF, LPF, and TPCCF. 

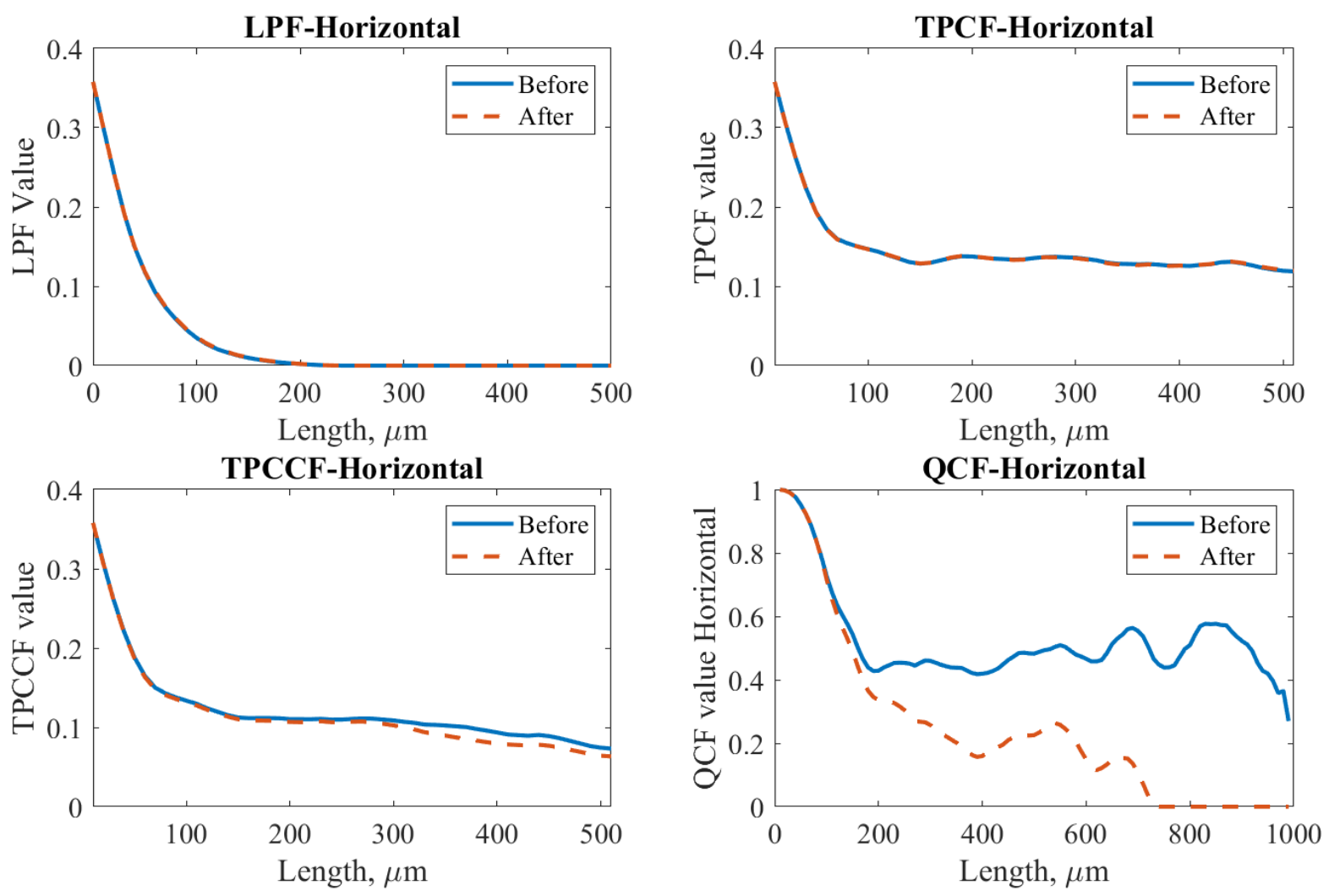

Fig. 11: Distributions of the four descriptors in horizontal direction before and after deletion of junction for Fig. 9c and Fig. 9d. For LPF, TPCF, and TPCCF, solid line shows the values before deletion of the junction, and dashed line shows the values after. For QCF, the values after deletion of junction are showed with colored lines.

Regarding the proposed application for the prediction of osteoporotic bone degradation, osteoclastic cells will resorb bone at increasing rate with age, leading mostly to the degradation of bone trabeculae and loss of their connections. This may lead to a sudden drop of the overall bone stiffness as described above and make it very fragile. This is largely unpredicted with classical imaging techniques measuring average bone density. Using classical descriptors will also not provide the necessary sensitivity for the small changes in the bone microstructure and loss of connections. On the contrary, QCF showed high sensitivity with significant changes assuming that image acquisition provides good knowledge of the microstructure distribution for QCF results analysis. This advantage can make QCF a useful descriptor for microstructure simulations and quantification of the large mechanical properties change due to small microstructure changes.

\section{Conclusion and future work}

A new statistical descriptor called quality of connection function (QCF) for the characterization and reconstruction of heterogeneous microstructures is presented. QCF shows a measure of the level of clustering within a given microstructure which was not visible when using other descriptors. Trabecular bone has been selected as a heterogeneous material sample with clustering. The new descriptor shows that, even with similar global or main cluster volume fraction, it is able to extract 
the differences from their specific microstructure distribution. It also shows that QCF value is an indicator of the corresponding stiffness of the material for a given microstructure distribution in a given direction. The new descriptor is tested on a number of samples and shows, on the contrary to other classical statistical descriptor, its capacity to extract the local connectivity variations, and hence potential important physical properties change. This descriptor is more useful when cluster connectivity is playing a significant role in overall physical properties. It proved to highlight specific sensitivity to microstructures distribution and connectivity while other descriptors were not. Future works include evaluating the capability of QCF to adequately reconstruct 3D microstructures accounting for the heterogeneous distribution. This work was started in Famouri et al. (2021), who showed that the microstructures chosen by QCF in the reconstruction process of 3D trabecular bone were more accurate compared to the one chosen by TPCF.

\section{Acknowledgements}

This work was supported by the Center for International Scientific Studies \& Collaboration (CISSC), Ministry of Science, Research \& Technology of Iran as well as the French Embassy in Iran through the French National Scientific Research Center (CNRS) and Campus France.

\section{References}

Abdel Rahman, R., et al.: An asymptotic method for the prediction of the anisotropic effective elastic properties of the cortical vein: superior sagittal sinus junction embedded within a homogenized cell element. J. Mech. Mat. Struc. 7(6), 593-611 (2012).

Bagherian, A., et al.: A novel numerical model for the prediction of patient-dependent bone density loss in microgravity based on micro-CT images. Cont. Mech. Thermo. 32, 927-943 (2019).

Baniassadi, M., et al.: Three-phase solid oxide fuel cell anode microstructure realization using twopoint correlation functions. Acta Materialia 59(1), 30-43 (2011a).

Baniassadi, M., et al.: New approximate solution for N-point correlation functions for heterogeneous materials. J. Mech. Phys. Sol. 60(1), 104-119, (2012).

Bazarin, R.L.M., et al.: Boundary Effects on the Tortuosity and Permeability of Idealized Porous Media. Transp Porous Med 136, 743-764 (2021).

Buryachenko, V.: Micromechanics of heterogeneous materials. Springer, ISBN 978-0-387-36827-6, (2007).

Jiao, Y., et al.: A Superior Descriptor of Random Textures and Its Predictive Capacity. Proc. Nat. Ac. Sc. 106, 17634 (2009).

Famouri, S., et al.: Refining Anticipation of Degraded bone microstructures during osteoporosis based on statistical homogenized reconstruction method via quality of connection function. Int. J. Comp. Mat. Sc. Eng. 9(4), 2050023 (2021).

Fullwood, D.T., et al.: Microstructure sensitive design for performance optimization. Prog. Mat. Sc. 55, 477-562 (2010).

Fullwood, D.T., et al.: A strong contrast homogenization formulation for multiphase anisotropic materials. J. Mech. Phys. Sol. 56(6), 2287-2297 (2008). 
Gerrard, D.D., et al.: Computational homology, connectedness, and structure-property relations. Comp. Mat. Cont. 15(2), 129-152 (2010).

Gigliotti, M., et al.: X-ray micro-computed-tomography characterization of cracks induced by thermal cycling in non-crimp 3D orthogonal woven composite materials with porosity. Composites Part A: App. Sc. Man. 112, 100-110 (2018).

Hamedani, H.A., et al.: Microstructure, property and processing relation in gradient porous cathode of solid oxide fuel cells using statistical continuum mechanics. J. Power Sources 196(15), 6325-6331 (2011).

Hasanabadi, A., et al.: 3D microstructural reconstruction of heterogeneous materials from 2D cross sections: a modified phase-recovery algorithm. Comp. Mat. Sc. 111, 107-115 (2016).

Hilfer, R.: Review on Scale Dependent Characterization of the Microstructure of Porous Media. Trans. Por. Med. 46, 373-390 (2002).

Izadi, H., et al.: Application of full set of two point correlation functions from a pair of 2D cut sections for 3D porous media reconstruction. J. Petrol. Sc. Eng. 149, 789-800 (2017).

Jiao, Y., et al.: Modeling heterogeneous materials via two-point correlation functions. II. Algorithmic details and applications. Physical Review E 77(3), 031135 (2008).

Kazempour, M., et al.: Numerical Simulation of Osteoporosis Degradation at Local Scale: A Preliminary Study on the Kinematic Loss of Mechanical Bone Stiffness and Microstructure. Biomed. Health Res., ISSN 0929-6, IOS Press 79, 86-93 (2019).

Lim, S., et al.: Pore structure refinement of cement paste incorporating nanosilica: study with dual beam scanning electron microscopy/focused ion beam (SEM/FIB). Mat. Charac. 145, 323-328 (2018).

Lu, B. and Torquato S.: Lineal-path function for random heterogeneous materials. Phys. Rev. A 45(2), 922 (1992).

Lu, B. and Torquato S.: Chord-length and free-path distribution functions for many-body systems. The J. Chem. Phys. 98(8), 6472-6482 (1993).

Madeo, A., et al.: Second-gradient models accounting for some effects of microstructure on remodelling of bones reconstructed with bioresorbable materials. Comp. Meth. Biomech. Biomed. Eng. 16(1), 260-261 (2013).

Marafani, E., et al.: Suitability of 2D modelling to evaluate flow properties in 3D porous media. Trans Por Med 134, 315-329 (2020).

Nikolov, S.: Revealing the Design Principles of High-Performance Biological Composites Using Ab initio and Multiscale Simulations: The Example of Lobster Cuticle. Adv. Mat. 22(4), 519-526 (2010).

Premakumar, P.: A*(A Star) search for path planning tutorial-File Exchange-MATLAB Central. Se. mathworks. Com (2016).

Rémond, Y., et al.: Applied RVE reconstruction and homogenization of heterogeneous materials. Wiley Online Library (2016).

Rezaei, F., et al.: The effectiveness of different thresholding techniques in segmenting micro CT images of porous carbonates to estimate porosity. J. Petrol. Sc. Eng. 177, 518-527 (2019). 
Scala, I., et al.: Mechanically-driven bone remodeling simulations : applications to LIPUS treated rat calvarial defects. Math. Mech. Sol. 22(10), 1976-1988 (2016).

Shanti, N.O., et al.: X-ray micro-computed tomography and tortuosity calculations of percolating pore networks. Acta Materialia 71, 126-135 (2014).

Sheidaei, A., et al.: 3-D microstructure reconstruction of polymer nano-composite using FIB-SEM and statistical correlation function. Comp. Sc. Tech. 80, 47-54 (2013).

Sheidaei, A., et al.: Influence of bone microstructure distribution on developed mechanical energy for bone remodeling using a statistical reconstruction method. Math. Mech. Sol. 24(10), 3027-3041 (2019).

Torquato, S.: Random heterogeneous materials: microstructure and macroscopic properties. Springer, Interdisciplinary Applied Mathematics, ISBN 0-387-95167-9, 16, (2002).

Torquato, S., et al.: Two-point cluster function for continuum percolation. The J. Chem. Phys. 88(10), 6540-6547 (1988). 